Relations industrielles

Industrial Relations

\title{
La crise du syndicalisme nord-américain: éléments d'interprétation
}

\section{Carla Lipsig-Mummé}

Volume 39, numéro 2, 1984

URI : https://id.erudit.org/iderudit/050028ar

DOI : https://doi.org/10.7202/050028ar

Aller au sommaire du numéro

Éditeur(s)

Département des relations industrielles de l'Université Laval

ISSN

0034-379X (imprimé)

1703-8138 (numérique)

Découvrir la revue

Citer cet article

Lipsig-Mummé, C. (1984). La crise du syndicalisme nord-américain: éléments d'interprétation. Relations industrielles / Industrial Relations, 39(2), 275-284. https://doi.org/10.7202/050028ar
Résumé de l'article

L'auteur explore deux avenues de ce qu'il faut déjà entrevoir comme la crise contemporaine du syndicalisme nord-américain.

htess//doi.org/10.7202/050028ar

Tous droits réservés @ C Département des relations industrielles de l'Université Laval, 1984
Ce document est protégé par la loi sur le droit d'auteur. L’utilisation des services d'Érudit (y compris la reproduction) est assujettie à sa politique d'utilisation que vous pouvez consulter en ligne.

https://apropos.erudit.org/fr/usagers/politique-dutilisation/ 


\title{
La crise du syndicalisme nord-américain éléments d'interprétation
}

\author{
Carla Lipsig-Mummé
}

\author{
L'auteur explore deux avenues de ce qu'il faut déjà entrevoir \\ comme la crise contemporaine du syndicalisme nord-américain.
}

Autant au Québec qu'au Canada, voire à travers toute l'Amérique du Nord, la dernière décennie fut loin d'être fructueuse pour les mouvements syndicaux. Peu importe leur tendance idéologique, soit vers la droite ou vers la gauche, les syndicats des secteurs publics et privés auront vécu l'expérience commune de la marginalisation de leur influence politique, ${ }^{1}$ de l'atténuation de leur pouvoir économique, de la baisse de leurs effectifs conjointement à l'aliénation croissante des membres restants, sans parler de la rétrogression des droits acquis allant parfois jusqu'à la dissolution de l'organisation syndicale. Pour la première fois depuis les années 1920, le rôle traditionnel du syndicat en tant que porte-parole des démunis est remis en cause. Partisans «d'un syndicalisme responsable» depuis une génération, c'est en pleine période de chômage structurel que les partis au pouvoir en Amérique du Nord ont décidé de déterrer la notion d'aristocratie ouvrière.

Il s'agit d'une vieille notion lourde d'implications lorsqu'elle est appliquée à l'ensemble de la classe ouvrière syndiquée, puisqu'elle incite la majorité non-syndiquée à chercher le blâme de la crise actuelle chez cette minorité syndiquée contre qui le pouvoir reproche tous les excès et tous les maux.

Ainsi, la déclaration suivante, issue de la bouche du député David Payne lors d'un conflit dans le secteur public au Québec, nous rappelle que

* LIPSIG-MUMMÉ, Carla, professeur, Université Concordia, Montréal.

1 La marginalisation politique des syndicats se manifeste d'abord en raison du fait que les partis politiques les plus pro-syndicaux ont perdu beaucoup d'influence aux États-Unis et au Canada anglais. Au Québec, malgré l'apparente remontée de l'influence syndicale au niveau politique il y a quelques années, le parti politique qui s'en disait responsable s'est de plus en plus dissocié de son «préjugé favorable aux travailleurs». En outre, la marginalisation politique se manifeste en raison du déclin quasi généralisé de l'influence de l'aile syndicale au coeur de partis comme le Nouveau Parti Démocratique et le Parti démocratique américain, au sein desquels cette faction syndicale avait pourtant joué un rôle de premier plan. 
l'attitude du président Regan envers les contrôleurs aériens (PATCO) en 1981 n'était pas uniquement le fruit de cet irréductible personnage, mais bien plus encore un signe des temps qui risque de se répéter tel un leitmotiv:

Il est proprement inacceptable qu'un groupe de salariés déjà privilégiés relativement à leur partenaire du secteur privé, prenne la population en otage et tente de forcer le gouvernement à leur verser la rançon de leur forfait. ${ }^{2}$

De leur rôle initial de porte-paroles des individus démunis et désavoués par cette société, les syndicats se sont vus ainsi transformés en bouc-émissaires, devenant les premiers responsables de la crise et se trouvant menacés par une campagne orchestrée pour déstabiliser les fondements mêmes du syndicalisme en Amérique du Nord ${ }^{3}$.

Pourquoi sommes-nous confrontés à un tel phénomène? Dans les pages qui suivent nous voulons explorer deux avenues de ce qu'il faut déjà entrevoir, sans dramatiser toutefois, comme la crise contemporaine du syndicalisme nord-américain. Dans un premier temps nous examinerons le contexte politico-économique: comment la réorganisation actuelle de la division internationale du travail (dont nous sommes loin d'avoir encore pesé les conséquences pour les industries et les syndicats des secteurs primaires et secondaires dans les pays développés) a suscité des pressions telles qu'elle a contribuée à une crise fiscale dans les pays les mieux nantis ainsi qu'une offensive concertée contre les syndicats dans ces pays. Dans un deuxième temps, nous explorerons deux causes importantes (et souvent négligées) de l'incapacité troublante des syndicats à articuler et à promouvoir une vision alternative à leur rôle légitime. La première est la dépendance institutionnelle du syndicat envers l'État. La deuxième cause réside dans un ensemble de présomptions relatives au rôle du capital et de l'État, acceptées pour ainsi dire presque inconsciemment par les syndicats nord-américains depuis la deuxième guerre mondiale et qui engendrent une résistance certaine à l'articulation de nouvelles stratégies.

\section{LE CONTEXTE POLITICO-ÉCONOMIQUE: LA CRISE DU SECTEUR PRIVÉ ET L'ÉTAT}

La crise économique actuelle nous apparaît à deux niveaux. Dans le secteur privé, elle apparaît comme une crise de rentabilité. Les grandes et moyennes compagnies dans les principales industries, comme celle de l'au-

2 David PAYNE, «Les limites du pouvoir: Une question de conscience», Québec, le 21 mars, 1983, p. 1 (dactylographié).

3 Or, cette campagne n'était pas limitée à l'Amérique đu nord: on la retrouve également en Angleterre et en Australie avant 1983. 
tomobile, ne sont plus concurrentielles avec le Japon et l'Allemagne fédérale, leur base technologique et leurs techniques productives étant parfaitement archaïques ${ }^{4}$. Afin de redevenir concurrentielles, ces industries ont besoin d'une réorganisation fondamentale autant au niveau de l'organisation du travail que de la technologie. Pour se faire, les sommes de capital requises sont colossales et il faut penser à une réduction importante de la main-d'oeuvre. Or, ces sommes ne sont pas aussi disponibles qu'elles étaient il y a dix ans, parce que les réseaux traditionnels de capitaux privés sont plus réticents envers des compagnies de moins en moins rentables. Les fusions d'entreprises géantes voilà quelques années ont rendu ce type de renflouage par les canaux privés un luxe du passé.

Comment arriver à moderniser dans de telles circonstances? L'itinéraire dans ce nouveau jeu est le suivant:

1. Obtenir les subventions requises afin de «robotiser» partiellement le travail à la chaîne. Les subventions, on le sait, c'est l'État qui les distribue.

2. Fermer les vieilles usines vétustes; en ouvrir de nouvelles qui ne pourront employer qu'une fraction des anciens employés, sans leurs droits traditionnels de sécurité d'emploi et d'ancienneté.

3. Se débarasser de plus de la moitié, et jusqu'à $80 \%$, de votre maind'oeuvre actuelle. Pour se faire, il faut la complicité, l'impuissance et la paresse du syndicat en place.

La crise apparaît également à un deuxième niveau, comme la crise fiscale de l'État ${ }^{5}$. Les États américain, québécois et canadien vivent le drame de la rentabilisation des compagnies privées comme provenant de deux demandes contradictoires. D'un côté, il y a les demandes pour des subventions massives. D'autre part, on exerce des pressions incessantes sur les programmes sociaux, pressions provenant du déluge de chômeurs, victimes de la restructuration du capital.

On s'en rend compte de plus en plus, les entreprises nord-américaines clament pour un État «super-dépanneur» afin de leur permettre de sortir de leur crise de non-rentabilité face à la concurrence internationale. Or, elles refusent de payer ces services, cherchant plutôt à imposer à l'État lui-même un modèle de rentabilité plus propre au secteur privé. D'autre part, l'État est sollicité par des victimes de cette restructuration du capital à l'échelle mondiale, des chômeurs permanents qui exigent plus qu'un dépannage tem-

4 Une remarquable mais courte historique du processus de «robotisation» dans l'industrie d'automobile et son impact sur l'emploi depuis 1950 est paru par Martin GLABERMAN dans Speaking Out, vol. 1, no 1, pp. 1-4 (n.d.).

5 Voir, à cet égard, le livre classique de James O'CONNOR, The Fiscal Crisis of the State, New York; St. Martin's Press, 1974. 
poraire face au nouvel ordre émergent. Ces victimes de la restructuration internationale exigent une nouvelle formation en fonction des emplois disponibles, un seuil décent du bien-être, l'accessibilité aux services médicaux, l'expansion de la construction pour des loyers à prix modiques, et un secours ou un répit pour les hypothèques mises en demeure.

Face à ces demandes et fidèles à l'idéologie émergente, les gouvernements nord-américains procèdent systématiquement aux coupures de services et au licenciement du personnel -- en somme, au démantèlement de «l'État-providence». D'autres options visant la création d'emplois, comme celles véhiculées par le nouveau gouvernement travailliste australien et les socialistes au pouvoir en France et en Grèce, ne sont même pas considérées.

Le trajet du démantèlement de l'État-providence mène à deux niveaux de confrontation avec les travailleurs syndiqués: les confrontations directes, à grand déploiement et particulièrement ressenties entre l'État et ses propres employés; et les confrontations indirectes, éparpillées et beaucoup moins visibles, entre les entrepreneurs que l'État cesse de subventionner ou de protéger et leurs anciens employés, victimes de la non-rentabilité sur le plan mondial.

Dans cette dernière catégorie, l'industrie secondaire semble avoir été plus sérieusement atteinte. Au Canada, dans les industries lourdes comme l'acier et l'automobile, de même que dans les industries plus traditionnelles que l'on surnomme les secteurs «mous», comme le vêtement et le textile, l'on prévoit une perte de l'ordre de $50 \%$ à $80 \%$ par rapport à la main-d'oeuvre actuelle d'ici les cinq prochaines années.

Pourtant, c'est surtout dans la première catégorie, à savoir les confrontations du secteur public, que l'on constate une situation des plus hétérogènes. Les «points chauds» se déplacent d'une région nord-américaine à une autre, selon le cadre juridique et la taille de l'unité syndicale en question.

Aux États-Unis, c'est d'abord au niveau municipal que se manifeste le drame des coupures de services, ainsi que leur corollaire, l'offensive menée contre les droits acquis des employés épargnés par les mises à pied. En fait, il faut reculer à l'année 1973 avant d'observer une augmentation des effectifs des gouvernements municipaux américains ${ }^{6}$. Entre 1973 et 1978 à New York, Rochester, Cincinnatti, Cleveland et Washington, D.C., les effectifs municipaux furent coupés de $10 \%$ à $20 \%{ }^{7}$. À Philadelphie, les enseignants ont mené une grève de deux mois en 1981, protestant la réouverture unilatérale de leur convention collective ${ }^{8}$. Mais tout comme pour les enseignants du

6 Carol O'CLEIREACAIN, «Going Public», Working Papers, janvier-février 1982, p. 12.

7 Ibid., p. 13.

8 Ibid. 
Québec, ce fut peine perdue. À travers les Etats-Unis, de nombreux syndicats ont du plier devant cette menace généralisée de faillite municipale, sacrifiant leurs augmentations salariales déjà négociées, perdant des centaines de postes.

Au Canada, les confrontations du secteur public se sont déroulées dans une arène plus vaste: soit au niveau des provinces ou du fédéral.

Mais dans l'ensemble nord-américain, les différents niveaux du pouvoir ont évidemment trouvé une première solution à leurs crises financières sur le dos des travailleurs syndiqués. En pleine crise, l'État-providence se transforme en État-matraque.

\section{LE RAPPORT ÉTAT-SYNDICAT}

Les mouvements syndicaux au Québec, au Canada et aux États-Unis nous semblent comme ayant été particulièrement passifs et divisés face à ce démantèlement de l'État-providence, à ce bouleversement technologique dans le secteur secondaire, au niveau de chômage qui s'annonce de plus en plus comme permanent, à leur nouveau rôle de bouc-émissaire. Pourquoi cette fragmentation, cette démobilisation? Comment expliquer une telle confusion, une telle impuissance? Mais avant tout, pourquoi cette absence de stratégie qui pourrait susciter une contre-offensive enrayant la perte des gains de toute une génération? Les indices d'affaiblissement contemporain des syndicats nord-américains se résument à cinq phénomènes. Premièrement, il y a l'accélération dans le rythme de la disparition d'emplois (soit au profit du Tiers-Monde, on en raison du chômage «technologique»); un nouveau rythme qui surpasse la création d'emplois à plein temps et qui entraîne la baisse des effectifs syndicaux au coeur même des lignes de force du syndicalisme traditionnel. Deuxièmement, il y a déclin du revenu réel: les syndicats se trouvant dans l'incapacité de négocier les clauses monétaires qui suivent l'inflation. Troisièmement, il y a la perte des droits acquis tels la sécurité d'emploi, l'ancienneté, le droit à la négociation collective, et le droit à l'emploi à temps plein. Quatrièmement, l'État se trouve soudainement justifié de contester la légitimité des conventions collectives par le biais de la réouverture unilatérale des conventions signées et par son refus de payer les augmentations déjà négociées. Même pour les conflits de travail les plus ordinaires, la régularité avec laquelle l'État est intervenu avec ses lois d'exception pour imposer ses propres conditions de travail a fait dire à deux commentateurs que nous étions entrés dans une «ère de l'exceptionnalisme permanente» ${ }^{9}$. Cinquièmement, il y a un éloignement progressif entre

9 L.V. PANITCH et D. SWARTZ, «From Free Collective Bargaining to Permanent Exceptionalism: The Economic Crisis and the Transformation of Industrial Relations in Canada», octobre 1982 (dactylographié). 
les syndiqués et leurs syndicats, phénomène qui aboutit dans certains cas à la désaffiliation ou à la désaccréditation; dans d'autres à une démobilisation répandue qui mine la vie syndicale. (Il est à noter que l'on retrouve parmi ces cinq indices d'affaiblissement, des phénomènes qui ne manifestent pas uniquement les nouveaux rapports de force issus de la crise, mais également les changements structurels ayant eux-mêmes engendrés cette crise.)

Pourtant, même en acceptant l'idée que le syndicat demeure au sein des sociétés capitalistes et, par sa nature même, un organisme dépendant plutôt réactif qu'initiateur, l'on se demande pourquoi les syndicats nord-américains semblent $s i$ incapables à réagir et à articuler une stratégie conséquente à ce nouveau défi ${ }^{10}$ ?

Car avant tout, la crise contemporaine du syndicalisme nord-américains en est une de désuétude stratégique. Afin de cerner l'étendue de ce problème, il faut d'abord examiner la situation telle qu'elle fut vécue pendant la longue période de prospérité qui débuta au lendemain de la deuxième guerre mondiale et se prolongea sans trop fluctuer jusqu'à la récession d'aujourd'hui.

Commençons par examiner les fondements de la stratégie syndicale au cours de ces trente années de prospérité.

Premièrement, peu importe leurs discours, les syndicats reconnaissaient l'entreprise privée comme le moteur de l'économie et la principale génératrice d'emplois. Les mouvements syndicaux de 1945 à 1975 ont tous confondu la croissance soutenue des profits à la croissance d'emplois, et par ce biais, à l'augmentation de leurs effectifs et à l'amélioration des conditions matérielles de leurs membres.

Liée à cette présomption d'une croissance illimitée, infinie, se trouvait une vision beaucoup plus large des possibilités de participation des syndiqués dans cette expansion du monde capitaliste. Nommé le modèle CIO, les syndicats industriels couplèrent leurs revendications monétaires au niveau relatif de croissance des profits des compagnies, présumant ainsi une croissance sans fin, un gâteau de plus en plus grand à se partager.

Ce faisant, ils négligèrent toute une série de questions absolument primordiale à la future vie syndicale, telles que le contrôle des changements dans les techniques de production et des priorités de recherche et de déve-

10 Comme l'écrivait le sociologue britannique V.L. ALLEN en 1971, «What they (unions) do is always in response to well-established forces such as rising prices, falling prices, unemployment, government action which influences living standards and over which they have little or no control... Trade unions are patently not initiators». The Sociology of Trade Unionism, Londres, Allen \& Unwin, 1971, p. 47. 
loppement, l'emplacement (ou le déplacement) des usines, l'investissement et le ré-investissement, etc. Il faut dire cependant que cette obsession étroitement «monétaire» des syndicats nord-américains n'était pas unique; les syndicats italiens ayant seuls entrepris sérieusement de négocier des questions mentionnées ci-haut.

Cette négligence fit son oeuvre et menotta rapidement les ouvriers syndiqués sur le plan économique. Dès 1950, les Travailleurs unis de l'automobile (TUA) concédaient la clause suivante à la General Motors:

the produets to be manufactured, the location of the plants, the schedules of the production, the methods, processes and means of manufacturing are solely and exclusively the responsability of the corporation. ${ }^{11}$.

Depuis lors, cette clause est devenue une clause type que l'on retrouve encore aujourd'hui dans la convention collective entre les TUA et la General Motors.

Et cette incapacité, voire ce manque d'intérêt à développer une stratégie d'ensemble qui articulerait les objectifs économiques avec tous ceux concernant l'organisation de la production, rend les syndicats nord-américains particulièrement vulnérables puisque c'est ce pouvoir unilatéral des entreprises qui leur permet de jongler avec le destin de centaines de milliers de syndiqués sous le couvert d'un vague projet de restructuration.

En deuxième lieu, depuis le début de l'État «providence», les syndicats nord-américains ont désiré que les partis successifs au pouvoir interviennent afin d'établir des seuils, de déterminer des normes minimales et de circonscrire des paramètres à partir desquels se déroulerait la confrontation, admettant ainsi leur impuissance, leur incapacité à régir cette question à la table de négociation ${ }^{12}$. Donc, jumelée à la reconnaissance que l'entreprise privée était le moteur de l'activité économique, l'on retrouvait l'image d'un État médiateur, d'un monde politique prêt à soutenir le syndicalisme minoritaire.

Il est étonnant d'observer que malgré le fossé idéologique séparant les centrales syndicales, comme par exemple l'AFL-CIO, la CTC et la CSN, la période qui s'écoule de 1945 à 1975 au Canada et aux États-Unis, et plus particulièrement de 1960 à 1975 au Québec, verra ces centrales partagées l'objectif commun (si peu élaboré soit-il) de l'élargissement du rôle de l'État, soit dans l'institutionalisation des conflits de travail, dans l'établisse-

11 Robert HOWARD, «Solidarity Begins at Home», Working Papers, janvier-février 1982, p. 21.

12 Comme, par exemple, le salaire minimum, l'assurance-chômage, l'indemnification des accidents du travail, les heures maximales, les programmes d'apprentissage subventionnés. 
ment de normes minimales pour les conditions de travail ou de vie, dans le maintien et la reproduction de la force de travail, ainsi que dans une intervention soutenue de planification économique.

Évidemment ces stratégies et les priorités visées par ces centrales auront énormément varié selon les secteurs, les régions, les périodes et ... les discours prononcés. Par exemple, le mouvement syndical américain a toujours joui de consensus lorsqu'il s'agissait d'accorder la priorité à la négociation collective et à son encadrement législatif. Or, les syndicats industriels de l'ancienne tradition CIO qui demeurent minoritaires au sein du mouvement syndical américain ont néanmoins privilégié eux aussi l'expansion du rôle de l'État pour la protection des groupes particulièrement exploités et en vue d'établir des seuils légaux pour les conditions de travail et d'existence. Ce dernier groupe de syndicats a vraiment cherché à accrô̂tre le rôle de l'État en matière de planification économique. Pour leur part, les syndicats de métier de la tradition AFL, demeuraient dans l'ensemble désintéressés par les problèmes des groupes syndiqués plus vulnérables et hostiles à la planification économique, sauf là où il s'agissait d'un protectionisme étroit.

Mais mises à part ces différences de priorités, les mouvements syndicaux nord-américains de la période de prospérité ont au moins partagé cette vision de dépendance envers un État que l'on voulait civilisateur des conflits de travail, médiateur principal, une sorte d'État-relais en somme.

La collaboration opportuniste entre l'État et le mouvement syndical et la propagation de l'idée de l'État-relais ont accéléré considérablement les deux changements structurels de la vie syndicale dans l'ère contemporaine: l'intégration $a ̀$ l'État et la dépendance face à l'État ${ }^{13}$. Par intégration, l'on entend le processus suivant lequel l'État s'immisce entre le capital et le travail afin de «résoudre» le conflit en s'attribuant les pouvoirs de l'un des partis opposés. L'on distinguera deux types d'intégration: l'intégration coercitive par l'entremise de quoi l'État s'approprie des pouvoirs syndicaux à l'encontre de la volonté syndicale; et l'intégration consensuelle où le syndicat collabore sciemment avec l'État dans l'aliénation d'une partie de ses pouvoirs, souvent en guise de marchandage pour un compromis à court terme, un projet ou une réforme sociale.

Ce processus d'intégration se joue de plus sur deux niveaux. À un premier niveau, l'État s'interpose intimement entre le syndicat et ses membres, obligeant le syndicat, par l'entremise du processus d'accréditation, à lui concéder le droit de parole au sein de ses propres membres. (Faut-il rap-

13 Pour une discussion de l'évolution historique de l'intégrationnisme au Quéec, voir C. LIPSIG-MUMMÉ, "Québec Unions and the State: Conflict and Dependence», dans Studies in Political Economy, vol. 1, no. 3, 1980, pp. 119-146. 
peler ici qu'un gouvernement qui se décerne le pouvoir d'annuler le droit de négocier à un syndicat, possède un moyen de pression très convaincant). En outre, les lois Landrum-Griffin aux États-Unis (1959), et les modifications apportées au Code du travail du Québec (1977) fournissent aux États respectifs le pouvoir de définir les procédures et pratiques électorales, l'admissibilité d'un syndiqué comme candidat à un poste syndical, une utilisation acceptable des cotisations syndicales et les limites d'une intervention dans le monde politique.

À un deuxième niveau, l'intégration des syndicats aux différents appareils étatiques et aux diverses institutions para-gouvernementales atteint une ampleur et une étendue insoupçonnées ${ }^{14}$. On pourrait en déceler les causes autant au niveau des exigences administratives des conventions collectives, qu'à celui de la participation croissante du leadership syndical au sein d'organismes politiques ou «para-politiques».

Avec le temps, l'intégration culmine dans une dépendance syndicale constante envers l'État et/ou l'employeur. En plus de participer aux organes et comités gouvernementaux, aux commissions d'enquête comme la Commission Cliche, les syndicats nord-américains sont devenus dépendants, pour leur fonctionnement, des subventions étatiques ou encore des ententes avec le patronat concernant la formation des ouvriers permanents, les programmes d'apprentissage, la publication et traduction des journaux syndicaux, les programmes de sécurité et de santé, la formation syndicale de base, les salaires des officiers élus et permanents, et, aux États-Unis, l'assurance-maladie.

Mais mise à part les dispositions concernant les services médicaux, les syndicats québécois reçoivent des subventions dans presque tous les autres domaines énumérés ici. Comparativement, les syndicats américains étant plus riches, ils demeurent plus autonomes sur le plan des subventions aux services syndicaux.

En résumé, quelque soit l'idéologie officielle des partis au pouvoir en cette période de récession et de crise, quels que soient leurs discours relativement au phénomène de décroissance vécu depuis une décennie, les États «providence» sont en voie de désintégration et de se transformer en Etats «matraques». Malgré cela, les mouvements syndicaux nord-américains continuent d'agir et d'évaluer selon les présomptions et les stratégies jugées convenables durant l'ère de prospérité ${ }^{15}$. Aujourd'hui, ces présomptions ne

14 On pourrait qualifier la collaboration entre la CSN et le gouvernement libéral de la Révolution tranquille d'une intégration consensuelle partielle. Pourrait-on en faire autant pour désigner le rapport actuel entre la FTQ et le gouvernement Lévesque?

15 Raymond SLIGER semble démontrer tout récemment la tenacité de ces présomptions, «Pour un syndicalisme responsable», Le Devoir, le 20 avril, 1983, p. 7. 
servent plus qu'à mystifier les syndicats. L'absence de toute stratégie relative à l'organisation de la production et à l'afflux d'une nouvelle technologie révolutionnaire mais avare de main-d'oeuvre, l'intégration et la dépendance financière des syndicats envers l'État et les employeurs, retardent singulièrement l'édification d'une nouvelle interprétation syndicale des nouveaux rapports de force. Loin de se précipiter vers la concertation, il est peut-être arrivé le temps pour le mouvement syndical de réévaluer les coûts réels de cette longue période d'intégration et des voies possibles vers une véritable autonomie syndicale.

\section{The Crisis of North American Unionism Elements and Interpretations}

The past decade has not been a propitious time for North American unions. Regardless of political ideology, public and private sector unions have alike experienced the attenuation of their political influence, a dramatic loss of membership, a growing passivity among the members who remain, and the undermining of acquired rights, sometimes to the point of union decertification. Union-busting has become a big business in the United States, while three out of five new unions formed in Canada choose independence rather than affiliation to an existing central. For the first time since the 1920 's, the principal political powers are replacing the notion of the union as spokesperson for the exploited by the value-laden concept of the union as a labor aristocracy.

What are the roots of this crisis of de-unionization? The paper looks first at the politic-economic context and argues that shifts occurring in the international division of labor have incited the major labor-intensive industries in North America to move against their unions by dramatic layoffs and cuts in benefits, at the same time as they pressure their states to reduce social supports for those who have joined the ranks of the structurally and permanently unemployed. The resulting fiscal crisis of the state has turned federal and state provincial governments, of whatever political coloration, to using their unions as scapegoats in the present crisis. «Scapegoat politics» in the industrial relations arena means cutting adrift minority unionism.

The second line of analysis looks at two reasons why North American unions have not been able to defend themselves and crystallize new strategies for their changed environment. The first of these reasons concerns the post-Depression institutional dependence of unions, regardless of their ideology, upon their respective states, including the assumption that states will establish for unions the whole gamut of threshold conditions that minority unionism cannot gain et the highly decentralized bargaining tables. The second reason for the present reactive confusion of North American unions resides in their generalized abdication of bargaining over technology and production decisions, an abdication which has left them terribly vulnerable to the ravages of layoffs and retooling.

The paper goes on to look at several dimensions of union dependence, through the notion of integration in relation to the state. It ends on a pessimistic note. 